\title{
Metodologias Analíticas para a Determinação de BiOMARCADORES DE FuMONISINAS EM AMOSTRAS BiolóGICAS
}

\author{
Celeste M. Lino*, Liliana J. G. Silva, Angelina Pena
}

As fumonisinas são micotoxinas que ocorrem a nivel mundial, principalmente em milho e seus derivados, e que podem afectar a saúde animal e humana. É assim essencial a análise de biomarcadores para avaliar a exposição animal e humana às referidas fumonisinas.

Este artigo revê as diferentes metodologias analiticas para a determinação destes biomarcadores, as próprias fumonisinas e o rácio esfinganina/esfingosina, em amostras biológicas, incluindo os diferentes procedimentos usados na extracção, purificação, derivatização, detecção e quantificação.

\section{INTRODUÇÃO}

As fumonisinas (FBs) são produzidas principalmente pelos fungos Fusarium verticillioides e Fusarium proliferatum [1]. Ambas as espécies são isoladas no milho, sendo a primeira a mais prevalecente [2].

Existem diferentes séries de FBs estruturalmente relacionadas [3]. No entanto, a que se encontra em maior abundância é a fumonisina $B_{1}\left(F B_{1}\right)$, sendo também a mais tóxica, seguindo-se a $\mathrm{FB}_{2}$ e a $\mathrm{FB}_{3}[4]$.

$A \mathrm{FB}_{1}$ é um diéster de propano-1,2,3ácido tricarboxílico e 2S-amino-12S, 16R-dimetilo-3S, 5R, 10R,14S,15R -pentahidroxieicoisano, em que os grupos hidroxilos em $\mathrm{C}_{14}$ e $\mathrm{C}_{15}$ estão esteri- ficados com um grupo carboxilo terminal do ácido tricarboxílico. $A \mathrm{FB}_{2}$ não possui o grupo hidroxilo em $C_{10}$, enquanto que a $\mathrm{FB}_{3}$ não apresenta o grupo hidroxilo em $C_{5}$ (Figura 1) [5].

As fumonisinas são moléculas fortemente polares, solúveis em água e acetonitrilo e insolúveis em solventes orgânicos [5]

A contaminação de alimentos e rações com FBs tem sido associada a doenças várias, quer em animais quer em humanos. Lino et al. [5], num artigo de revisão, aludiram a estas patologias, sendo frequentemente citadas, entre outras [6-8], a leucoencefalomalacia equídea (ELEM), edema pulmonar (PPE) em suínos e a toxicidade renal em diferentes espécies. Têm sido epidemiologicamente relacionadas com o cancro esofágico (EC) em humanos [9] e são suspeitas do aumento da incidência de defeitos no tubo neural (NTD) [10]. Com base nos dados actuais, a Agência Internacional de Pesquisa sobre o Cancro classificou a $\mathrm{FB}_{1}$ como possivelmente carcinogénica para o homem, incluindo-a no grupo 2B [11].

Uma forma de avaliar a exposição de populações às FBs consiste em calcular a respectiva ingestão diária estimada (EDI), através dos níveis de fumonisinas ingeridos nos distintos itens alimentares. Outra consiste em avaliar os níveis de FBs ou de outros biomarcadores seguros, como a proporção esfinganina/esfingosina (Sa/So) em amostras biológicas [12-15] uma vez que estas micotoxinas interferem no metabolismo dos esfingolípidos [5, 12] (Figura 2).<smiles>[R3]C(CCCCC([R1])CC([R2])C(C)N)CC(C)CC(OC(=O)CC(CC(=O)O)CC(=O)O)C(OC(=O)CC(CC(=O)O)C(=O)O)C(C)CCCC</smiles>

Fumonisina $\mathrm{B} 1$ : $\mathrm{R} 1=\mathrm{OH}$; $\mathrm{R} 2=\mathrm{OH}$; $\mathrm{R} 3=\mathrm{OH}$; Fumonisina B2: $\mathrm{R} 1=\mathrm{OH} ; \mathrm{R} 2=\mathrm{OH} ; \mathrm{R} 3=\mathrm{H}$ Fumonisina B3: R1 $=\mathrm{H} ; \mathrm{R} 2=\mathrm{OH} ; \mathrm{R} 3=\mathrm{OH}$;

Figura 1 Fórmula estrutural das fumonisinas $B_{1}-B_{3}$

\footnotetext{
Grupo de Bromatologia - Centro de Estudos Farmacêuticos, - Faculdade de Farmácia, Universidade de Coimbra, 3000-295 Coimbra, Portugal cmlino@ci.uc.pt
}

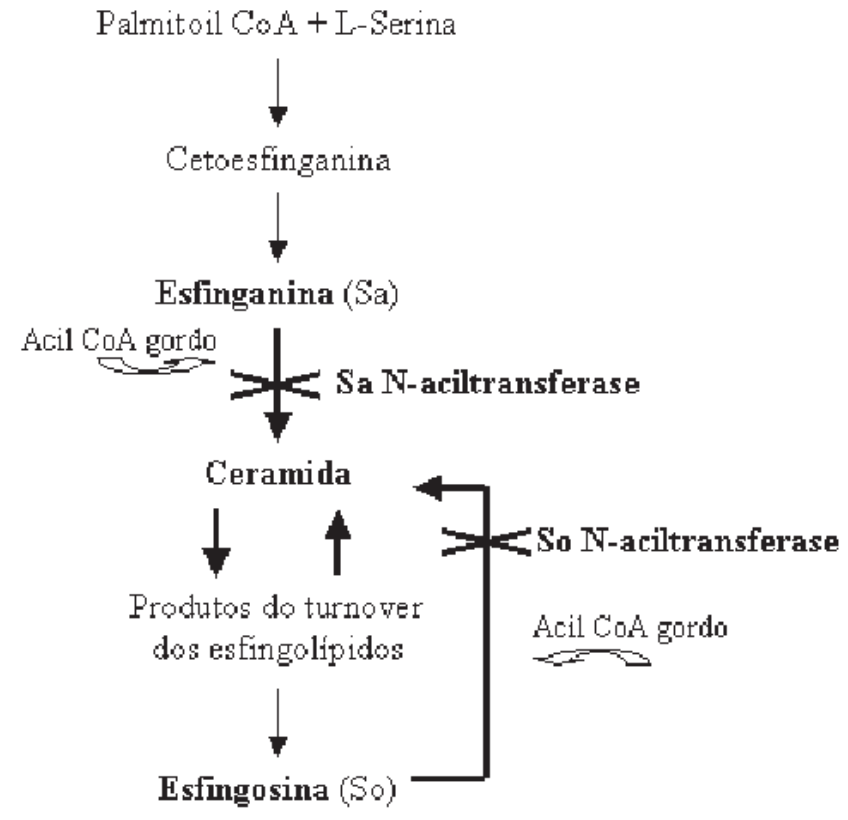

Figura 2 Modo de acção das fumonisinas 
Apesar dos resultados obtidos a partir do cálculo da ingestão diária serem úteis numa primeira etapa, estes revelam-se pouco precisos porque são baseados na análise de alguns alimentos e em tabelas relativas ao consumo através da dieta [16].

Os níveis reduzidos de FBs no plasma e o seu rápido declínio após a respectiva administração, bem como o insucesso de todos os estudos em detectar quantidades significativas de metabolitos circulantes, mostram que aqueles não são adequados para serem utilizados como biomarcadores na exposição de animais a estas toxinas. Contudo, a observação de que a $F B_{1}$ e a $F B_{2}$ inibem a enzima $\mathrm{N}$-aciltransferase na biossíntese de novo de esfingolípidos, levando a uma acumulação de esfinganina, uma base esfingóide, e a um aumento do rácio esfinganina-esfingosina sugere que esta proporção é um biomarcador com potencial e preferencial relativamente às $\mathrm{FBs}[5,17]$. Consequentemente torna-se fundamental desenvolver métodos eficientes para monitorizar a exposição humana com o uso de biomarcadores apropriados de forma a garantir a saúde humana $[18,19]$.

$\mathrm{Na}$ determinação de FBs em amostras biológicas, a grande maioria dos métodos referenciados envolve extracção, recorrendo a misturas de solventes polares, seguida de um processo de purificação com extracção em fase sólida (SPE) com colunas de fase reversa $\left(C_{18}\right)[19,20]$, cartuchas de troca iónica forte (SAX) $[21,22]$ e com resina polimérica não iónica Amberlite XAD-2 em mistura com SAX [13]. Na análise de Sa e de So as amostras são sujeitas a extracção, seguida ou não de um processo de purificação com extracção em fase sólida (SPE) com sílica gel $[23,14]$.

Após derivatização com ortoftaldeído (OPA), usado na maior parte dos casos [14, 21-23], ou outro agente de derivatização como o naftaleno-2,3-dicarboxialdeído (NDA) [24], a detecção e quantificação de ambos os biomarcadores são efectuadas recorrendo, principalmente, a mé- todos cromatográficos, sendo a cromatografia líquida (LC) com detecção fluorimétrica (LC-FD) [17, 19, 21, 23, 25] a mais utilizada. A detecção por espectrometria de massa (LC-MS) [9, 22] tem como vantagem não requerer derivatização. A cromatografia em camada fina (TLC) [19], os métodos imuno-histológicos [26] e os radioactivos [18] são também reportados, embora menos usados.

Neste artigo são abordadas diferentes metodologias analíticas bem estabelecidas e validadas, aplicadas à extracção, purificação, derivatização, detecção, quantificação e confirmação de FBs e esfinganina-esfingosina em amostras biológicas.

\section{Metodologias analíticas para a DETERMINAÇÃO DE FUMONISINAS}

Em 1992, Shephard et al. [21] reportaram pela primeira vez um método para a determinação da $\mathrm{FB}_{1}$ em fluídos biológicos, plasma e urina, de ratos macho BDIX. Até esta data a metodologia analítica usada na determinação da $\mathrm{FB}_{1}$ restringia-se à sua quantificação em materiais de cultura, milho, rações e alimentos à base de milho.

\section{ExtRACÇÃo}

Em virtude de se tratar de moléculas fortemente polares, as FBs são solúveis em água e solventes polares e insolúveis em solventes orgânicos [27]. Na extracção, nomeadamente para a desproteinização, os solventes de eleição são o metanol $(\mathrm{MeOH})$ [9, $21,22,28]$ e o acetonitrilo (ACN) [9, 20]. Na remoção de lípidos recorre-se à utilização de hexano [9, 22].

Chelule et al. [19] determinaram a presença de $\mathrm{FB}_{1}$ em fezes humanas, procedendo à extracção com EDTA, acidificação e centrifugação.

Os métodos analíticos previamente desenvolvidos para a determinação de $\mathrm{FB}_{1}$ em amostras fisiológicas foram modificados por Shephard et al. [20] para tornar possível a determinação de $F_{2}$. Esse objectivo foi atingido pelo uso de solventes de extracção menos polares uma vez que esta micotoxina apresenta uma polaridade inferior à da $\mathrm{FB}_{1}$.

Tal como a $\mathrm{FB}_{1}$, a $\mathrm{FB}_{2}$ é fortemente retida na matriz fecal e apenas por repetições sucessivas do processo extractivo é possível uma adequada recuperação analítica. O metanol simples não é indicado para ser utilizado como solvente de extracção na análise desta matriz, aumentando-se a eficiência extractiva com a utilização da mistura EDTA-MeOH [20]. Também a extracção de FBs a partir da matriz cabelo humano é complexa e morosa, para além de exigir pré-tratamento exaustivo da referida matriz [22].

\section{Purificação}

A purificação é conseguida recorrendo à extracção em fase sólida (SPE) em fase reversa, usando sílica octadeciligada $\left(C_{18}\right)[17,19,20,28]$ ou troca aniónica (SAX) $[9,13,17,21$, 22,28 ] e ainda Amberlite XAD-2 [13].

Shephard et al. [21] efectuaram a purificação de urina e de plasma de ratos macho BDIX para determinar a presença de $\mathrm{FB}_{1}$ com SPE, usando cartuchas SAX acondicionadas com $\mathrm{MeOH}$ e $\mathrm{MeOH}-\mathrm{H}_{2} \mathrm{O}$, sendo a eluição efectuada com ácido acético $\left(\mathrm{CH}_{3} \mathrm{COOH}\right)$ em $\mathrm{MeOH}$. Após evaporação o resíduo redissolve-se em $\mathrm{MeOH}$. Segundo estes autores, a urina contém elevadas concentrações de interferentes, o que obsta a purificação com cartuchas SAX. Assim, para obter recuperações e reprodutibilidade aceitáveis, pequenos volumes de urina devem ser diluídos diversas vezes antes de se iniciar a fase de purificação. Shephard et al. [20] procederam de forma semelhante na determinação de $\mathrm{FB}_{2}$ em amostras animais, no entanto, com excepção da urina, o resíduo foi redissolvido em borato de sódio.

Shetty e Bhat [13] defendem que o método de Shephard et al. [21] não pode ser aplicado na análise de urina humana devido ao elevado número de interferentes, tendo usado um método mais sensível para determinar $\mathrm{FB}_{1}$ neste fluído biológico. Para ultrapassar este inconveniente, o processo de purificação foi realizado em duas 
etapas, com resina Amberlite XAD-2 e com cartuchas SAX, o que permitiu a ausência de picos interferentes aquando da análise cromatográfica sem comprometer a recuperação da $\mathrm{FB}_{1}$. Estes autores defendem que, através da aplicação deste método, é possível detectar quantidades diminutas (limite de detecção, LOD, inferior a $8 \mathrm{ng} / \mathrm{mL}$ ) de FBs em urina de indivíduos que consomem uma dieta contaminada.

As cartuchas SAX foram também escolhidas por Sewram et al. [22] na análise de FBs em cabelo humano, por Meyer et al. [9] na análise de tecidos de suínos, por Shephard e Snijman [17] na purificação de extractos de plasma e urina e por Sewram et al. [28] em extractos de amostras de pêlo de macacos vervet. Estes autores recorreram também à utilização de colunas $\mathrm{C}_{18}$. Na purificação de extractos fecais, quer aquando da análise de $\mathrm{FB}_{1}$ ou de $\mathrm{FB}_{2}$ verifica-se a utilização de colunas $C_{18}[17,19,20]$.

$\mathrm{Na}$ Tabela 1 encontram-se sumariadas as condições cromatográficas utilizadas pelos vários autores.

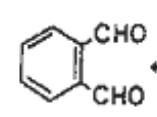

(A)

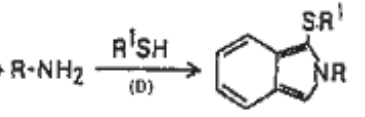

(B)

(C)
Figura 3 Reacção de derivatização com OPA (A: OPA; B: fumonisinas; C: derivado OPA; D: MCE)

Shephard et al. [20, 21] realizaram uma derivatização pré-coluna adicionando $200 \mu$ l de OPA a $50 \mu$ l de resíduo redissolvido. O derivado OPA apresentou-se estável durante 4 minutos. Este método já havia sido usado na determinação de $\mathrm{FB}_{1}$ em rações à base de milho. Assim, a utilização de OPA foi investigada com o objectivo de desenvolver uma metodologia analítica para quantificar $\mathrm{FB}_{1}$ em amostras fisiológicas, tendo sido bem conseguida a separação do derivado OPA/FB . .

\section{Detecção E Quantificação}

Tal como na determinação de fumonisinas em milho e em alimentos à base de milho [30], a cromatografia de alta eficácia (LC) é a técnica de separação analítica para a determinação de $\mathrm{FB}_{1}$ em amostras biológicas de rato usando colunas de sílica octaligada $\left(\mathrm{C}_{8}\right)$. Outras colunas analíticas de fase reversa, como $\mathrm{C}_{18}$ têm sido usadas mais recentemente $[9,17,19,20,22,29]$.

Na maioria dos casos é usada eluição isocrática, sendo a fase móvel mais recorrente a mistura $\mathrm{MeOH}-\mathrm{NaH}_{2} \mathrm{PO}_{4}$ [17, 19-21, 29]. Sewram et al. [22] e Meyer et al. [9] usaram eluição em gradiente. Assim, os primeiros autores, fizeram variar ao longo do tempo da corrida a proporção de $\mathrm{H}_{2} \mathrm{O}-\mathrm{ACN}$-ácido fórmico. Os segundos autores empregaram os mesmos solventes, mas isoladamente e em mistura: $\mathrm{A}$ : $\mathrm{ACN}$; B: $\mathrm{H}_{2} \mathrm{O} ; \mathrm{C}: \mathrm{H}_{2} \mathrm{O}$-ácido fórmico.

Como as FBs apresentam um comportamento iónico em solução, as separações em colunas de fase reversa são baseadas numa mistura de mecanismos de fase reversa e de troca iónica. Observa-se uma grande variação de selectividade entre as diferentes colunas. As FBs não eluem convenientemente da maior parte das

Tabela 1 Condições cromatográficas por LC para a determinação de bases esfingóides em amostras biológicas

\begin{tabular}{|c|c|c|c|c|c|c|c|c|c|c|}
\hline \multicolumn{4}{|c|}{ Coluna analítica } & \multicolumn{2}{|l|}{ Fase Móvel } & \multirow{2}{*}{$\begin{array}{l}\text { Fluxo } \\
\left(\mathrm{mLmin}^{-1}\right)\end{array}$} & \multirow{2}{*}{$\begin{array}{l}\lambda(\mathrm{nm}) \\
\mathrm{OPA}\end{array}$} & \multirow{2}{*}{$\begin{array}{l}\text { Gama } \\
\text { linearidade }\end{array}$} & \multirow{2}{*}{$\begin{array}{l}\text { Limite de } \\
\text { deteç̧ăo }\end{array}$} & \multirow[b]{2}{*}{ Referéncia } \\
\hline Tipo & $\begin{array}{l}\text { Comprimento } \\
(\mathrm{cm})\end{array}$ & $\begin{array}{l}\text { d.i. } \\
(\mathrm{mm})\end{array}$ & $\begin{array}{l}\text { Tamanho da } \\
\text { partícula }(\mu \mathrm{m})\end{array}$ & Eluiçăo isocrática & Eluição em gradiente & & & & & \\
\hline $\mathrm{RP}-\mathrm{C}_{8}$ & 12,5 & 4,0 & 5 & $\begin{array}{l}\mathrm{MeOH}: \mathrm{NaH}_{2} \mathrm{PO}_{4} 0,1 \mathrm{M} \\
(66: 34) \text { ajustado a pH } 3,4 \\
\text { com } \mathrm{H}_{3} \mathrm{PO}_{4}\end{array}$ & & 1 & $\begin{array}{l}\text { Exc: } 335 \\
\text { Em:440 }\end{array}$ & - & $50 \mathrm{ng} / \mathrm{mL}$ & [21] \\
\hline RP-C 18 & & 4,0 & 3 & $\begin{array}{l}\mathrm{MeOH}: \mathrm{NaH}_{2} \mathrm{PO}_{4} 0,1 \mathrm{M} \\
(72-28) \text { ajustado a pH } 3,4 \\
\text { com } \mathrm{H}_{3} \mathrm{PO}_{4}\end{array}$ & & 1 & $\begin{array}{l}\text { Exc: } 335 \\
\text { Em:440 }\end{array}$ & - & - & {$[17,20,29]$} \\
\hline$R P-C_{18}$ & 15 & 3,8 & & $\begin{array}{l}\text { ACN: } \mathrm{H}_{2} \mathrm{O}: \mathrm{CH}_{3} \mathrm{COOH} \\
(50: 50: 1)\end{array}$ & & 1 & $\begin{array}{l}\text { Exc: } 335 \\
\text { Em: } 440\end{array}$ & - & $<8 \mathrm{ng} / \mathrm{mL}$ & [13] \\
\hline$R P-C_{18}$ & 15 & 3,9 & & $\begin{array}{l}\mathrm{MeOH}: \mathrm{NaH}_{2} \mathrm{PO}_{4} 0,1 \mathrm{M} \\
(80-20) \text { ajustado a pH } 3,4 \\
\text { com } \mathrm{H}_{3} \mathrm{PO}_{4}\end{array}$ & & 1 & $\begin{array}{l}\text { Exc: } 335 \\
\text { Em:440 }\end{array}$ & - & $50 \mathrm{ng} / \mathrm{g}$ & [19] \\
\hline RP-C 18 & 15 & 4,6 & 5 & & $\begin{array}{l}\text { A- } \mathrm{H}_{2} \mathrm{O}: \mathrm{ACN}-\hat{A}_{\mathrm{C}} \text {. fórmico } \\
(90: 10: 0,1) \\
\mathrm{B}-\mathrm{H}_{2} \mathrm{O}: \mathrm{ACN}-\mathrm{Ac}_{\mathrm{c}} \text { fórmico } \\
(10: 90-0,1)\end{array}$ & 0,7 & & $L O Q-100 \mu g / L$ & $25 \mathrm{pg}$ & [22] \\
\hline$R P-C_{18}$ & 15 & 2,1 & 3,5 & & $\begin{array}{l}\text { A-ACN } \\
\text { B- } \mathrm{H}_{2} \mathrm{O} \\
\text { C- } \mathrm{H}_{2} \mathrm{O}: \text { Ácido fórmico }(85: 5)\end{array}$ & 0,2 & & - & $\begin{array}{l}\text { ND a } 2 \\
\mu g / \mathrm{kg}\end{array}$ & [9] \\
\hline
\end{tabular}

- Informação não disponível

\section{Derivatização}

O reagente de derivatização eleito na análise de $\mathrm{FB}_{1}$ e de $\mathrm{FB}_{2}$ em amostras fisiológicas é o ortoftaldeído (OPA) $[13,17,19-21,29]$. A reacção de derivatização dá-se entre o reagente derivatizante e a amina primária das fumonisinas, na presença de 2-mercaptoetanol (MCE) (Figura 3). mais usada na determinação destas micotoxinas em amostras biológicas.

As fumonisinas, moléculas polares, solúveis em água e em solventes polares, são ideais para a detecção e a quantificação por LC em fase reversa. Em 1992, Shephard et al. [21] reportaram, pela primeira vez, metodologia colunas de fase reversa quando são injectadas com sistemas de solventes neutros e não tamponizados. Para se conseguirem melhores resultados a fase móvel deve ser acídica, o que se consegue através da adição de ácido acético ou fórmico à fase móvel ou pelo uso de um tampão volátil [30]. A LC-FD é amplamente usada na aná- 
lise de FBs em alimentos para consumo humano e animal, sendo também amplamente usada na análise das mesmas em amostras biológicas. A grande parte dos investigadores recorre ao detector de fluorescência, estabelecendo como comprimento de onda de excitação $\left(\lambda_{\text {exc }}\right) 335 \mathrm{~nm}$ e como comprimento de onda de emissão $\left(\lambda_{\text {em }}\right) 440 n m$, uma vez que o agente de derivatização escolhido é o OPA [19-21, 29].

A cromatografia líquida acoplada à espectrometria de massa (LC-MS) permite a análise directa e a confirmação das FBs [31]. Sewram et al. [22] e Meyer et al. [9] usaram a interface electro-spray na análise de FBs. Sewram et al. [22] optimizaram os parâmetros MS separadamente para a $\mathrm{FB}_{1}$, $\mathrm{FB}_{2}$ e $\mathrm{FB}_{3}$. Uma energia de colisão de $32 \%$ foi usada para fragmentar os iões moleculares protonados e os iões resultantes de cada fumonisina foram monitorizados e usados como indicadores da presença destas micotoxinas em cabelo humano. A aplicação de LC-MS permitiu incrementar a especificidade da detecção.

No estudo de Meyer et al. [9] a FB foi fragmentada e monitorizada tendo a sua identificação e quantificação sido feita com base nos tempos de retenção e área dos picos dos iões seleccionados ( $\mathrm{m} / \mathrm{z} 722,388,300)$ por comparação com um padrão externo.

A aplicação de técnicas de espectrometria de massa permite a obtenção de limites de detecção (LODs) na ordem dos $25 \mathrm{pg}$ em amostras de cabelo humano [22], $1 \mu \mathrm{g} / \mathrm{kg}$ em tecido muscular e adiposo de suínos e $2 \mu \mathrm{g} / \mathrm{kg}$ em tecido cardíaco de suínos [9]. Os autores que aplicaram detecção por fluorescência na análise de FBs em amostras biológicas reportaram LODs na ordem dos $50 \mathrm{ng} / \mathrm{mL}$ para urina de ratos macho BDIX [21], $8 \mathrm{ng} / \mathrm{mL}$ para urina humana [13] e $0,05 \mu \mathrm{g} / \mathrm{kg}$ para fezes humanas [19].

Chelule et al. [19] também analisaram a presença de $\mathrm{FB}_{1}$ em fezes humanas por cromatografia em camada fina (TLC) em placa de sílica. A dissolução dos extractos secos foi efectuada com $\mathrm{ACN}-\mathrm{H}_{2} \mathrm{O}$, o desenvolvimento com butanol- $\mathrm{H}_{2} \mathrm{O}-\mathrm{CH}_{3} \mathrm{COOH}$ e a revelação por pulverização com anisaldeído. A purificação insuficiente com $\mathrm{C}_{18}$ levou ao aparecimento de interferências. O facto de algumas amostras serem positivas quando analisadas com TLC e negativas quando analisadas com LC, pode dever-se ao anisaldeído que não é específico para as FBs.

Também Shephard et al. [20] usaram TLC para analisar a pureza radio-química da ${ }^{14} \mathrm{C}-\mathrm{FB}_{2}$ isolada a partir de culturas de F. moniliforme marcadas com carbono radioactivo.

Em 1996, Prelusky et al. [18] aplicaram métodos radioactivos para o estudo da distribuição de resíduos de derivados $-{ }^{14} \mathrm{C}$ em amostras biológicas de suínos Yorkshire alimentados com $\mathrm{FB}_{1}$ marcada. Para medir a radioactividade das amostras, estas foram analisadas através dum procedimento de combustão de oxigénio. As medições feitas por contagem de cintilação líquida (LSC) foram corrigidas para eficiência de combustão, diluição, extinção, fundo e eficiência de contagem. O limite de detecção em tecidos foi de aproximadamente $46 \mathrm{ng} / \mathrm{g}$.

As características hidrofílicas das FBs conduzem à sua rápida excreção dos tecidos animais. Métodos mais sensíveis são necessários para a visualização directa de FBs in situ. Uma técnica capaz de identificar as FBs antes da sua excreção seria útil para a sua detecção em tecidos e poderia explicar a origem de algumas lesões. Alguns métodos imuno-histoquímicos têm sido avaliados. A técnica da avidina-biotina-peroxidase consiste numa combinação de técnicas anatómicas, imunológicas e histoquímicas que permite a localização de antigénios específicos em estruturas de tecidos definidas. Foi desenvolvida uma reacção específica entre a $\mathrm{FB}_{1}$-anticorpo monoclonal e o sistema avidina-biotina-peroxidase para demonstrar a presença de FBs em células hepáticas e renais de aves, tendo permitido melhorar o diagnóstico patológico de lesões agudas e subagudas [26]. Através da utilização de anticorpos monoclonais anti-FB ${ }_{1}$ e do sistema avidina-biotina-peroxidase, o fígado e rins de frangos Leghorn brancos, aos quais foi administrada $\mathrm{FB}_{1}$ e $\mathrm{FB}_{2}$, foram analisados por Buim et al. [26] com o objectivo de detectar e conhecer a distribuição de FBs. Desta reacção resulta um complexo electronodenso que pode ser analisado ao microscópio óptico ou electrónico.

\section{Metodologias analíticas para de- TERMINAÇÃo DA PROPORÇÃo Sa/So}

A medição directa de FBs em amostras biológicas não é um bom indicador da exposição, nomeadamente humana, às FBs uma vez que estas micotoxinas são eliminadas pouco tempo após a ingestão [32]. A estrutura química das FBs assemelha-se largamente com a da esfingosina e a da esfinganina (Figura 4), inibindo a enzima ceramida sintetase, uma enzima chave no metabolismo dos esfingolípidos, levando à acumulação de esfinganina e à redução dos níveis de esfingolípidos complexos [23].

\section{Extracção}

Em 1994, Riley et al. [23] reportaram, pela primeira vez, metodologia analítica complexa e morosa [14] para a determinação do rácio $\mathrm{Sa} / \mathrm{So}$ a partir de soro, urina e tecidos animais diversos. Após extracção com $\mathrm{CHCl}_{3}-\mathrm{MeOH}$, incubação a $37^{\circ} \mathrm{C}$, adição de $\mathrm{CHCl}_{3}$ e $\mathrm{H}_{2} \mathrm{O}$ alcalinizada e centrifugação, a fase orgânica, após secagem e evaporação, é submetida a um processo de hidrólise alcalina com o objectivo de clivar os acilglicerolípidos e hidrolisar os lisoesfingolípidos libertando, assim, a esfingosina. A detecção de Sa livre é complexa já que a eficiência extractiva por vezes é baixa, podendo esta limitação ser problemática quando o seu conteúdo é da ordem de $\mathrm{pmol} / \mathrm{g}$, observando-se também variações intra e interindividuais na quantificação da Sa e da So. Nesta situação a utilização do padrão interno Sa C:20 simplifica a quantificação. O seu uso é, no entanto, desnecessário quando, para se detectar a inibição da Sa N-aciltransferase induzida pelas FBs, se utiliza a elevação do rácio Sa/So e não as bases esfingóides isoladamente [14, 23, 25, 33].

A metodologia de Riley et al. [23] foi seguida por outros autores [32-36], embora com algumas modificações, 
nomeadamente no que diz respeito à adição de $\mathrm{NH}_{4} \mathrm{OH}$ à amostra [14, 32] .

Numa tentativa de melhoramento do processo anterior, alguns autores optaram pelo recurso à hidrólise ácida com a finalidade de libertar as bases esfingóides a partir dos esfingolípidos complexos [37].

Na determinação de Sa e So em soro de ratos BDIV e de humanos alguns autores optam pelo acetato de etilo $[38,39]$ que permitiu reduzir o tempo dispendido no processo extractivo e obtenção de recuperações mais elevadas. Não obstante, a sensibilidade deste método na análise de soro humano não é completamente satisfatória [38].
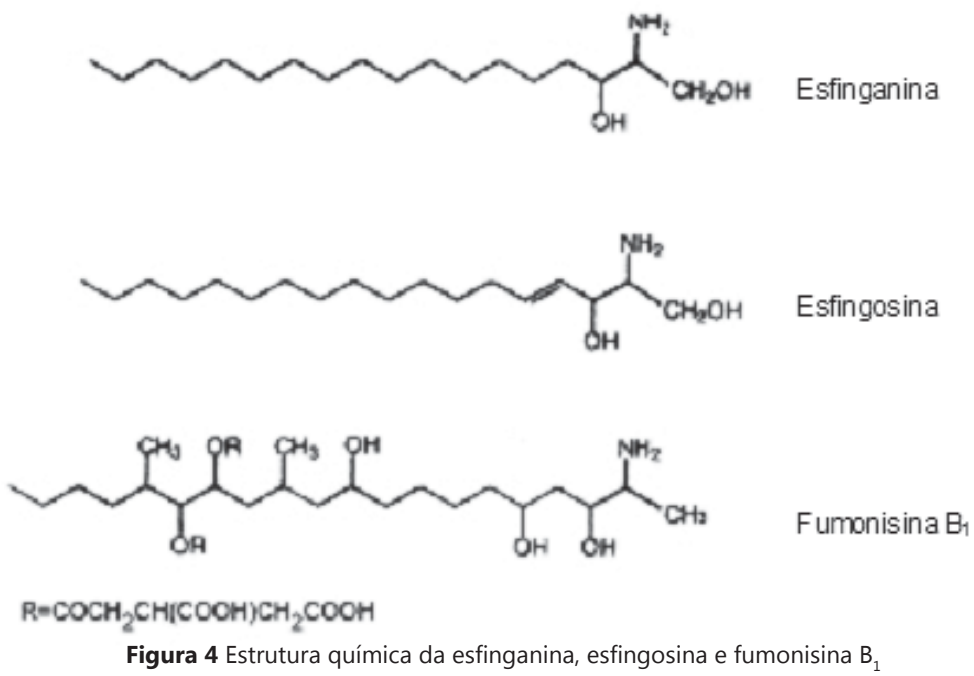

Shephard e Westhuizen [40] adaptaram o método que Solfrizzo et al. [14] usaram para a determinação do rácio Sa/So na urina, para procederem à análise no soro, defendendo que a determinação de bases esfingóides nesta matriz permite ultrapassar o obstáculo levantado pelos baixos níveis de Sa presentes na urina de indivíduos do género masculino. Após desproteinização do soro com $\mathrm{MeOH}$, centrifugação, mistura com $\mathrm{H}_{2} \mathrm{O}$ e $\mathrm{NH}_{4} \mathrm{OH}$, a extracção foi efectuada com $\mathrm{CHCl}_{3}$. Esta metodologia foi seguida na análise do rácio $\mathrm{Sa} / \mathrm{So}$ em plasma e urina de populações rurais da África do Sul expostas a FBs [41].

Para obviar o problema das baixas concentrações de Sa livre na urina nos indivíduos do género masculino $[14,39]$, o que inevitavelmente origina baixas sensibilidades, recorre-se ao aumento do volume da amostra. Assim, Qui e Liu [25] e Garren et al. [39] extraíram $2 \mathrm{~mL}$ de urina de indivíduos do género feminino e 40-60 $\mathrm{mL}$ de urina de indivíduos do género masculino.

\section{Purificação}

Agrande maioria dos autores não recorre à purificação dos extractos [23-25, 32-35, 37, 38, 39, 42]. Porém outros optam por SPE com uma minicoluna de sílica gel $[14,43]$, que permite reduzir o tempo de análise comparativamente com os que recorrem à hidrólise alcalina. A eluição das bases esfingóides é efectuada com $\mathrm{CHCl}_{3}-\mathrm{MeOH}-\mathrm{NH}_{4} \mathrm{OH}$ e o resíduo foi redissolvido em $\mathrm{MeOH}-\mathrm{H}_{2} \mathrm{O}$. 
formando-se um derivado 1-ciano-2-alquil-benzoisoindole ( $\mathrm{CBI}$ ), uma estrutura altamente fluorescente (Figura 5). Comparativamente à derivatização com OPA, a fluorescência dos derivados OPA diminui 36,8\% [24]. Situação análoga foi verificada por Lino et al. [44] na análise de amostras de milho e seus derivados. A intensidade da fluorescência, apresentada pelo NDA é também maior [24].
No que concerne às colunas analíticas, todos os procedimentos para LC utilizam colunas de fase reversa $C_{18}$ para separar as bases esfingóides, já que estas permitem uma eficiência elevada na análise dos derivados OPA, permitindo também uma pressão reduzida e boa estabilidade [14]. Em relação à escolha das dimensões da coluna observa-se alguma disparidade (Tabela 2).

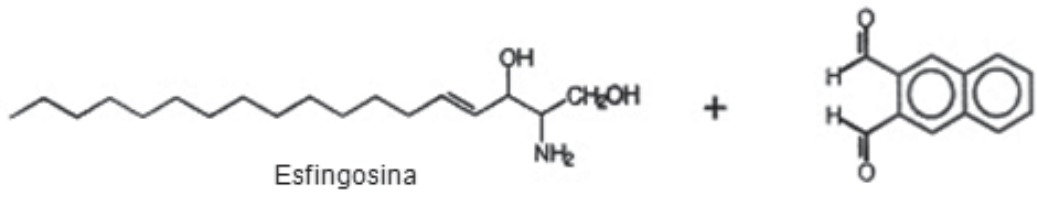

NDA

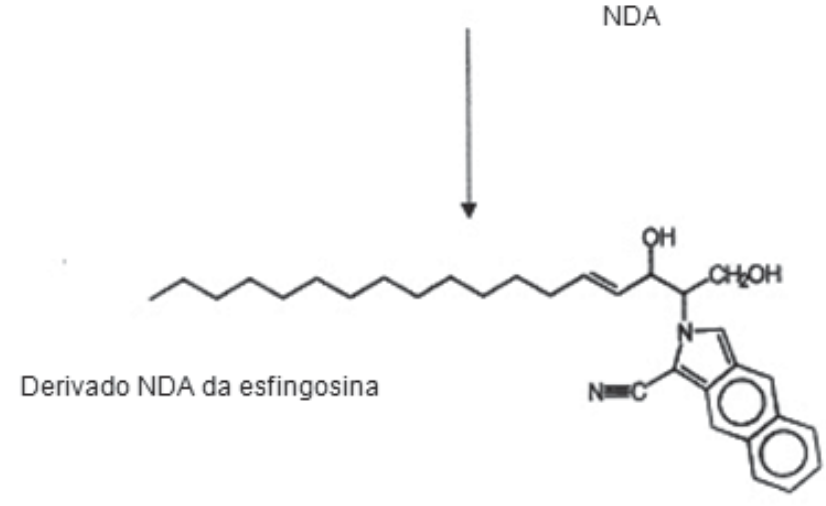

Figura 5 Reacção de derivatização com NDA

\section{Detecção e quantificação}

A cromatografia líquida ( $L C$ ) é a técnica analítica de separação mais usada. A razão desta popularidade, além de residir na sua elevada sensibilidade, na sua capacidade para efectuar determinações precisas e de separar espécies não voláteis, deve-se sobretudo ao facto de possuir uma grande aplicabilidade a substâncias com interesse primordial para a indústria e na investigação científica [45].

Em 1991, Wang et al. [42] reportaram, pela primeira vez, o mecanismo pelo qual as FBs inibem a biossíntese de novo dos esfingolípidos. O uso de LC permitiu analisar a massa das bases de cadeia longa.

A cromatografia líquida com detecção por fluorescência (LC-FD) tem sido usada por inúmeros investigadores para determinar a quantidade relativa de So e Sa livres no soro, urina e tecidos variados $[14,23,25,32,33,37$, 38, 39, 41-43].
Dois tipos de eluição são usados, isocrática [23, 24, 32-34, 37, 40, 41] e gradiente [14, 25, 35, 38, 39, 43]. Em ambas as eluições o solvente mais utilizado é o metanol, verificando-se, no entanto, variações nas misturas utilizadas (Tabela 2). Outros autores recorrem ao acetonitrilo [24]. Solfrizzo et al. [14] não registaram diferenças nos cromatogramas usando uma fase móvel em cuja composição entrava ou não tampão fosfato de potássio. Consideram que este tampão, usado por Riley et al. [23], entre outros autores, não é necessário já que os derivados OPA-Sa e OPA-So não possuem grupos ionizáveis, ao contrário do que sucede com as FBs.

A lavagem da coluna com ACN [33] ou $\mathrm{MeOH}$ [14] entre cada injecção é importante para impedir a eluição de picos interferentes.

Os fluxos adoptados variam, sendo o mais utilizado o de $1 \mathrm{ml} / \mathrm{min}$. O mesmo acontece com os comprimentos de onda de excitação $\left(\lambda_{\text {exc }}\right)$ e de emissão $\left(\lambda_{\text {em }}\right)$ que variam de acordo com o reagente de derivatização empregue (Tabela 2).
Diversos métodos de LC-FD estão disponíveis para a análise de Sa e de So. No entanto, as interferências provenientes dos diferentes componentes das amostras dificultam uma quantificação precisa e exacta das bases esfingóides. Adicionalmente, estes procedimentos são morosos e complexos, requerendo a execução de ínumeros passos [46].

A espectrometria de massa (LC-MS) é uma ferramenta eficiente permitindo elevadas selectividade e sensibilidade [45], tendo também bastante aplicabilidade na análise de FBs [12, 31, 47, 48].

Seefelder et al. [45] reportaram metodologia analítica rápida e precisa para a determinação de Sa e de So em culturas celulares do epitélio renal humano usando cromatografia líquida com ionização por electrospray e detecção por espectrometria de massa (LC-ESI-MS). A separação cromatográfica foi realizada numa coluna analítica de fase reversa $\mathrm{C}_{18}$ usando gradiente binário de ácido trifluroacético (TFA) em $\mathrm{H}_{2} \mathrm{O}$ e de TFA em $\mathrm{MeOH}$ (fluxo de $200 \mu \mathrm{L} / \mathrm{min}$ ). A ionização por electrospray foi efectuada com uma voltagem de $3,5 \mathrm{kV}$ e a $200^{\circ} \mathrm{C}$, tendo o $\mathrm{N}_{2}$ sido usado como sheath e como gás auxiliar. O detector de massa modo selected ion monitoring (SIM), detectou iões positivos $[\mathrm{M}+\mathrm{H}]^{+}$de So $(\mathrm{m} / \mathrm{z}$ 300), Sa ( $m / z$ 302) e PSO ( $m / z 318)$. A aplicação deste método permitiu um LOD de 10 pg para a So e de 25 pg para a Sa.

A cromatografia em camada fina (TLC) e métodos radioactivos têm sido usados. Através destas técnicas foi possível verificar que a disrupção do metabolismo dos esfingolípidos seria um evento crítico em doenças associadas ao consumo de FBs [42]. Na análise de lípidos as células expostas a FBs durante 0-4 dias foram depois colocadas num meio com $\left[{ }^{14} \mathrm{C}\right]$ serina na presença ou ausência de FBs. Após incubação de 2 a 16 horas, as células foram retiradas do meio e os lípidos extraídos e sujeitos a uma hidrólise ácida. A Sa e a So resultantes foram então separadas por TLC em placas de sílica gel, desenvolvidas com $\mathrm{CHCl}_{3}-\mathrm{MeOH}-\mathrm{NH}_{4} \mathrm{OH}$, e o perfil radioactivo determinado por 
Tabela 2 Condições cromatográficas por LC para a determinação de bases esfingóides em amostras biológicas

\begin{tabular}{|c|c|c|c|c|c|c|c|c|c|c|c|}
\hline \multicolumn{4}{|c|}{ Coluna Analítica } & \multicolumn{2}{|l|}{ Fasemóvel } & \multirow{2}{*}{$\begin{array}{l}\text { Fluxo } \\
\left(\mathrm{mL} \min ^{-1}\right)\end{array}$} & \multicolumn{2}{|l|}{$\lambda(\mathrm{nm})$} & \multirow{2}{*}{$\begin{array}{l}\text { Gama de } \\
\text { linearidade }\end{array}$} & \multirow{2}{*}{$\begin{array}{l}\text { Limite de } \\
\text { detecção }\end{array}$} & \multirow{2}{*}{ Referência } \\
\hline Tipo & $\begin{array}{l}\text { Comprimento } \\
(\mathrm{cm})\end{array}$ & $\begin{array}{l}\text { di. } \\
(\mathrm{mm})\end{array}$ & $\begin{array}{l}\text { Tamanho } \\
\text { particula } \\
(\mu \mathrm{m})\end{array}$ & Eluição isocrática & Eluição gradiente & & OPA & NDA & & & \\
\hline RP-C 18 & 10 & 8 & 4 & $\begin{array}{l}\mathrm{MeOH}(89-90 \%) \text {.tampão } \\
\text { fosfato potássio } 0,005 \mathrm{M} \text { (10- } \\
11 \%), \mathrm{pH} 7,0 \\
\end{array}$ & & 2 & $\begin{array}{l}\text { Exc: } 335-340 \\
\text { Em: } 440-255\end{array}$ & & - & - & {$[23,37]$} \\
\hline RP-C 18 & 10 & 8 & 4 & $\begin{array}{l}\text { MeOH: tampão fosfato potássio } \\
(0,005 \mathrm{M})(93: 7) \mathrm{pH} 7,0\end{array}$ & & 2 & $\begin{array}{l}\text { Exc: } 335-340 \\
\text { Em: } 440-255\end{array}$ & & - & - & [33] \\
\hline RP-C 18 & 15 & 4,6 & 5 & & $\begin{array}{l}\mathrm{A}-\mathrm{MeOH}: \mathrm{H}_{2} \mathrm{O}(9: 1), 13 \mathrm{~min} \\
\mathrm{~B}-\mathrm{MeOH}, 5 \mathrm{~min}\end{array}$ & 1 & $\begin{array}{l}\text { Exc: } 335 \\
\text { Em: } 440\end{array}$ & & - & - & {$[14,43]$} \\
\hline RP-C 18 & 25 & 4,6 & 5 & & $\begin{array}{l}\mathrm{A}-\mathrm{K}_{2} \mathrm{HPO}_{4}(0,07 \mathrm{M}): \mathrm{MeOH} \\
(1-9) \\
\text { B-MeOH }\end{array}$ & 1 & $\begin{array}{l}\text { Exc: } 340 \\
\text { Em: } 355\end{array}$ & & - & - & {$[38]$} \\
\hline RP-C 18 & 15 & 4,6 & 5 & $\mathrm{MeOH} \cdot \mathrm{H}_{2} \mathrm{O}(90: 10)$ & & 1,5 & $\begin{array}{l}\text { Exc: } 340 \\
\text { Em: } 355\end{array}$ & & - & 0,02 (rácio) & {$[40]$} \\
\hline RP-C 18 & 15 & 4,6 & 5 & $\mathrm{MeOH}: \mathrm{H}_{2} \mathrm{O}(91-9)$ & & 1 & $\begin{array}{l}\text { Exc: } 340 \\
\text { Em: } 355\end{array}$ & & - & - & [41] \\
\hline RP-C 18 & & & & $\begin{array}{l}\text { MeOH.tampão fosfato potássio } \\
(5 \mathrm{mM})(91: 9), \mathrm{pH} 7,4\end{array}$ & & & & & - & - & {$[34]$} \\
\hline $\mathrm{RP}-\mathrm{C}_{18}$ & & & & $\mathrm{MeOH}: \mathrm{H}_{2} \mathrm{O}(91 \cdot 9)$ & & 2 & & & - & - & {$[32,36]$} \\
\hline RP-C 18 & 15 & 3,9 & 4 & & $\begin{array}{l}\mathrm{A}-0,07 \mathrm{M} \mathrm{K}_{2} \mathrm{HPO}_{4}-\mathrm{MeOH} \\
(1-9) \\
\text { B-MeOH }\end{array}$ & 1 & $\begin{array}{l}\text { Exc: } 335 \\
\text { Em: } 440\end{array}$ & & 0,1 a $0,3 \mathrm{ng}$ & $\begin{array}{l}\text { 우: } \\
0,15 \mathrm{ng} / \mathrm{mL} \\
8: \\
0,005 \mathrm{ng} / \mathrm{mL}\end{array}$ & {$[25]$} \\
\hline RP-C 18 & 15 & 4,6 & & $\begin{array}{l}\text { ACN: } \mathrm{H}_{2} \mathrm{O}(87 \%) \\
\text { ACN: } \mathrm{H}_{2} \mathrm{O}(90 \%) \\
\end{array}$ & & 1 & $\begin{array}{l}\text { Exc:340 } \\
\text { Em:455 }\end{array}$ & $\begin{array}{l}\text { Exc:252 } \\
\text { Em:483 }\end{array}$ & $0,1 \mathrm{pmol}$ & 10 a 50 pmol & {$[24]$} \\
\hline RP-C 18 & 25 & 4,6 & 5 & & $\begin{array}{l}\mathrm{A}-\mathrm{K}_{2} \mathrm{HPO}_{4}(0,07 \mathrm{M}): \\
\mathrm{MeOH}(1: 9) \\
\text { B-MeOH }\end{array}$ & 1 & $\begin{array}{l}\text { Exc: } 335 \\
\text { Em: } 440\end{array}$ & & - & - & [39] \\
\hline
\end{tabular}

scanning radiométrico e contagem de cintilação, sendo as bases esfingóides visualizadas com ninhidrina.

$\mathrm{Na}$ medição da actividade relativa da So $\mathrm{N}$-acetiltransferase em hepatócitos intactos as células foram incubadas com $\mathrm{FB}_{1}$ e depois com [3H]-So. Os lípidos foram então extraídos e as $[3 \mathrm{H}]$-ceramidas da $[3 \mathrm{H}]$-So foram separadas usando TLC com placas de sílica desenvolvidas em éter dietílico-MeOH e visualizadas com vapor de iodo e a quantidade de material marcado determinada por contagem de cintilação [42]

Os resultados obtidos por Yoo et al. [37] foram confirmados por TLC. Assim, os lípidos obtidos na hidrólise ácida e não derivatizados foram separados em placas de sílica gel desenvolvidas com $\mathrm{CHCl}_{3}-\mathrm{MeOH}-\mathrm{NH}_{4} \mathrm{OH}$ e as bases $\mathrm{Sa}$ e So visualizadas recorrendo a vaporização com ninhidrina.

\section{ConcLusões}

Métodos sensíveis e confiáveis para a determinação de fumonisinas e do rácio $\mathrm{Sa} /$ So, biomarcadores para as fumonisinas em diferentes matrizes biológicas, são de absoluta necessidade para avaliar o grau de exposição humana e animal.

Atendendo à complexidade das técnicas extractivas e de purificação disponíveis, são desejáveis outras incursões no sentido de aumentar a especificidade e, simultaneamente, minorar os custos e a morosidade.

De entre os vários métodos abordados neste artigo, a LC com derivatização pré-coluna e detecção fluorimétrica é mais amplamente utilizada na determinação e quantificação de fumonisinas.

Espera-se que, com o desenvolvimento científico, a LC-MS se torne numa técnica mais sensível, menos onerosa e, em consequência, mais disponível para efectuar estes estudos, já que permite a análise directa de extractos, sem recurso à derivatização, e a respectiva confirmação. Espera-se ainda que a metodologia permita cada vez mais uma quantificação precisa e exacta de biomarcadores para que a avaliação da exposição seja o mais fiável possível.

\section{Agradecimentos}

Os autores agradecem à FCT e POCTI (FEDER) a bolsa de doutoramento SFRH/BD/19382/2004 concedida a Liliana J. G. Silva.

\section{REFERÊNCIAS}

[1] C.P. Woloshuk, W. Shim, Appl. Environ. Microbiol. 67 (2001) 1607-1612.
[2] P.M. Scott, G. A. Lawrence, J. FoodProtection 58 (1995) 1379-1382.

[3] J.-A. Seo, Y.-W.Lee, Appl. Environ. Microbiol. 65 (1999) 1331-1334.

[4] R. Labuda, D. Tančinova, K. Hudec, Ann. Agric. Environ. Med. 10 (2003) 61-66.

[5] C.M. Lino, L.J.G. Silva, A.S. Pena, Rev. Port. Ciênc. Vet. 99 (2004) 181-192.

[6] K.A. Voss, R.T. Riley, W.P. Norred, C.W. Bacon, F.I. Meredith, P.C. Howard, R.D. Plattner, T.F.X. Collins, D.K.Hansen, J.K. Porter, Environ. Health. Perspectives 109 (suppl. 2) (2001) 259-266.

[7] M.R. Carratù, T. Cassano, A. Coluccia, P. Borracci, V. Cuomo, Toxicology Letters 140-141 (2003) 459--463.

[8] E.E. Creppy, P. Chiarappa, I. Baudrimont, P. Borracci, S. Moukha, M.R. Carratú, Toxicology 201 (2004) 115-123.

[9] K. Meyer, K. Mohr, J. Bauer, P. Horn, M. Kovács, Food Add. Contam. 20 (2003) 639-647.

[10] M.E. Stack, J. AOAC Int. 81 (1998) 737-740.

[11] IARC (International Agency For Research On Cancer) in IARC Monographs on the evaluation of carcinogenic risks to humans 82 (2002) 301-366. IARC, Lyon, France.

[12] T. Cirillo, A. Ritieni, F. Galvano, R.A. Cocchieri, Food Add. Contam. 20 (2003) 566-571.

[13] P.H. Shetty, R.V. Bhat, J. Chromatography B 705 (1998) 171-173.

[14] M. Solfrizzo, G. Avantaggiato, A. Visconti, J. Chromatography B 692 (1997) 87-93.

[15] P.C. Turner, P. Nikiema, C.P.Wild, $\mathrm{Mu}$ tation Research 443 (1999) 81-93. 
[16] H. Crews, G. Alink, R. Andersen, V. Braesco, B. Holst, G. Maiani, L. Ovesen, M. Scotter, M. Solfrizzo, R. van den Berg, H. Verhagen, G. Williamson, British Journal of Nutrition $\mathbf{8 6}$ (suppl. 1) (2001) s5-s35.

[17] G.S. Shephard, P.W. Snijman, Food Chem. Toxicol. 37 (1999) 111-116.

[18] D.B. Prelusky, J.D. Miller, H.L. Trenholm, Food Add. Contam. 13 (1996) 155-162.

[19] P.K. Chelule, N. Gqaleni, A. A. Chuturgoon, M.F. Dutton, Biomarkers 5 (2000) $1-8$

[20] G.S. Shephard, P.G. Thiel, E.W. Sydenham, J. Chromatography A 692 (1995) 39-43.

[21] G.S. Shephard, P.G. Thiel, E.W. Sydenham, J. Chromatography $\mathbf{5 7 4}$ (1992) 299-304

[22] V. Sewram, N. Mshicileli, G.S. Shephard, W.F.O. Marasas, Biomarkers 8 (2003) 110-118.

[23] R.T. Riley, E. Wang, A.H. Merrill, J. AOAC Int. 77 (1994) 533-540.

[24] Y.-H. Cho, H.-S. Yoo, J.-K. Min, E.-Y. Lee, S.-P. Hong, Y.-B. Chung, Y.-M. Lee, J. Chromatography A 977 (2002) 69-76.

[25] M. Qiu, X. Liu, Food Add. Contam. 18 (2001), 263-269.

[26] M.R. Buim, A.P.F.R.L. Bracarense, I.G. Guimarães, O. Kawamura, Y. Ueno, E.Y. Hirooka, Nat. Toxins 7 (1999) 279-282
[27] C.R. Pozzi, J.R.P. Arcaro, I.A. Júnior, H. Fagundes, B. Corrêa, Ciência Rural 32 (2002) 901-907

[28] V. Sewram, J.J. Nair, T.W. Nieuwoudt, W.C. Gelderblom, W.F. Marasas, G.S. Shephard, J. Anal. Toxicol. 25 (2001) 450-455

[29] G.S. Shephard, P.G. Thiel, E.W. Sydenham, P.W. Snijman, Food.Chem. Toxic. 33 (1995) 591-595.

[30] R.D. Plattner, Nat. Toxins 7 (1999) 365--370.

[31] W. Xie, C.J. Mirocha, J. Chen, J. Agric. Food Chem. 45 (1997) 1251-1255.

[32] S. Ribar, M. Mesarié, M. Baumen, J. Chromatography B 754 (2001) 511-519 .

[33] G.S. Shephard, L. Westhuizen, P.G. Thiel, W.C.A.; Gelderblom, W.F.O. Marasas, D.J. Schalkwyk, Toxicon 34 (1996) 527-534.

[34] E. Wang, W.P. Norred, R.T. Riley, F.I. Meredith, A.H.Jr. Merril, J. Nutrition 129 (1999) 214-220.

[35] C.C. Abnet, C.B. Borkowf, Y.-L. Qiao, P.S. Albert, E. Wang, A.H. Merril, S.D. Mark, Z.-W. Dong, P.R. Taylor, S.M. Dawsey, Cancer Causes and Control 12 (2001) 821-828.

[36] S.T. Tran, J.D. Bailly, D. Tardicu, S. Durand, G. Benard, P. Guerre, ChemicoBiological Interactions 146 (2003) 6172.

[37] H.-S. Yoo, W.P. Norred, R.T. Riley, Toxicology in vitro 10 (1996) 77-84.
[38] M. Castegnaro, L. Garren, D. Galendo, W.C.A. Gelderblom, P. Chedule, M.F. Dutton, C. P. Wild, J. Chromatography B 720 (1998) 15-24.

[39] L. Garren, D. Galendo, C.P. Wild, M. Castegnaro, Food Add. Contam. 18 (2001) 850-856.

[40] G.S. Shephard, L.Westhuizen, J. Chromatography B 710 (1998) 219-222.

[41] L. Westhuizen, N.L. Brown, W.F.O. Marasas, S. Swanevelder, G.S. Shephard, Food Chem. Toxicol. 37 (1999) 1153-1158.

[42] E. Wang, W.P. Norred, C.W. Bacon, R.T. Riley, A.H.Jr. Merril, The Journal of Biological Chemistry 266 (1991) 14486-14490.

[43] M. Solfrizzo, S.N. Chulze, C. Mallmann, A. Visconti, A. Girolamo, F. Rojo, A. Torres, Food Add. Contam. 21 (2004) 1090-1095

[44] C.M. Lino, L.J.G. Silva, A.S. Pena, Anal. Bioanl. Chem. 384 (2006) 1214-1220.

[45] D.A. Skoog, F.J. Holler, T.A. Nieman, In Principles of Instrumental Analysis 5th; Brace College Publishers; Hartcourt, Philadelphia, 1998, p.725.

[46] W. Seefelder, G. Schwerdt, R. Freudinger, M. Glekle, H.-U. Humpt, J. Chromatography B 780 (2002) 137$-144$.

[47] S.M. Musser, R.D. Plattner, J. Agric. Food Chem. 45 (1997) 1169--1173.

[48] D. Royer, H.-U. Humpf, P.A. Guy, Food Add. Contam. 21 (2004) 678-692.

\section{CURIOSIDADES HISTÓRICAS}

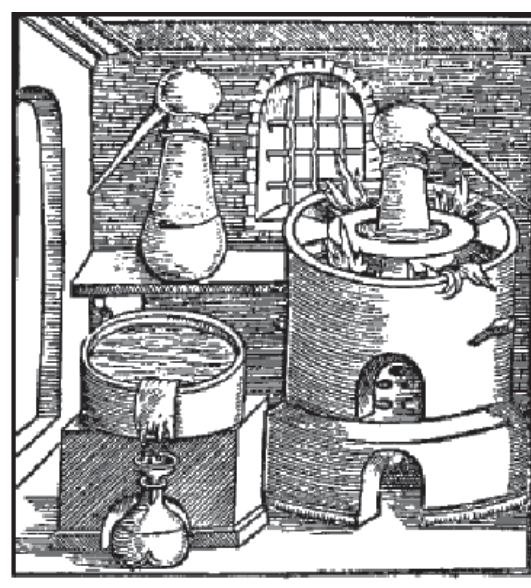

Destilaçäo, Alchemiae Gebri Arabis ..., 154

«A coisa primordial na alquimia é que se pode realizar trabalhos práticos e conduzir experiências, quem não faz trabalhos práticos nem faz experiências nunca dominará a alquimia».

Jābir ibn Hayyān, conhecido pelo seu nome latinizado Geber, o pai da alquimia árabe, escreveu 22 tratados alquímicos. Desenvolveu métodos básicos que descreveu nos seus livros, nomeadamente técnicas de purificação e separação como fusão, sublimação, cristalização fraccionada, filtração - destilação sem fogo ou destillatio per filtrum - calcinação e destilação (simples, fraccionada e por arrastamento de vapor). Desenvolveu ainda vários instrumentos para o seu trabalho alquímico, nomeadamente o alambique com que destilou o espírito do vinho, o al kohl.

Ao estudar várias reacções químicas notou que numa reacção química estão envolvidas quantidades bem determinadas dos reagentes. A descoberta dos ácidos cloridrico e nítrico é atribuída por muitos historiadores a Geber que os teria descoberto destilando vários sais na presença de ácido sulfúrico - Zayt al-Zaj, ou óleo de vi- tríolo. A ele também é creditada a descoberta do ácido cítrico (existente em limões e outros citrinos), ácido acético (vinagre), ácido tartárico (do resíduo de fabricação de vinho), o uso de dióxido de manganês na fabricação de vidro - um processo ainda hoje utilizado - e a introdução de termos químicos como ál-cali (alcalino).

$\mathrm{Na}$ Idade Média, os seus tratados de alquimia, como o Kitab al-Kimya, o Livro da Composição Alquímica, traduzido por Robert de Chester em 1144, e o Kitab as-Sab'een introduzido por Gerard de Cremona nos finais do século XII, influenciaram marcadamente os alquimistas europeus. Marcelin Berthelot traduziu ainda outros livros de Jābir ibn Hayyān sob os títulos de Livro do Reino, Livro das Balanças e Livro do Mercúrio Oriental. 